\title{
Ex Vivo Induction of mRNA in Human Whole Blood as a New Platform of Drug and Dietary Supplement Development
}

\author{
Masato Mitsuhashi, ${ }^{1,2,6}$ Mieko Ogura, ${ }^{1}$ Katsuya Endo, ${ }^{2}$ Kazuhiko Obara, ${ }^{2}$ Hiroshi Izutsu, ${ }^{2}$ Stephan R. Targan, ${ }^{3}$ \\ Motoko Maemura, ${ }^{4}$ Daisuke Tachikawa, ${ }^{4}$ and Atsushi Shinagawa ${ }^{5}$
}

Received July 16, 2007; accepted November 21, 2007; published online January 9, 2008

\begin{abstract}
Purpose. We introduced a new concept of ex vivo gene expression analysis (Mitsuhashi, Clin Chem 53:148-149, 2007), where drug action was simulated under physiological conditions. This model system was applied to study various fields of drug development.

Materials and Methods. Heparinized human whole blood was incubated with drugs for less than $4 \mathrm{~h}$. The changes of specific mRNA were then quantified using the method we developed (Mitsuhashi, Tomozawa, Endo, and Shinagawa, Clin Chem 52:634-642, 2006).

Results. The mRNA quantitation method was used as a model system to study the following areas: (1) identification of respondents and non-respondents, (2) ex vivo compound screening, (3) determination of individually optimized doses, (4) drug-to-drug comparison, (5) assessment of leukocyte toxicity, (6) discovery of molecular targets, (7) assessment of the action of dietary supplements, and (8) characterization of respondents and non-respondents for various dietary supplements.

Conclusion. Since ex vivo assays are safe, a large number of healthy donors and disease patients can be recruited to identify individual-to-individual variations, which is not available from current preclinical study models. Although each system should be validated using a large number of samples, the ex vivo analysis will be a new tool for the development of drugs and dietary supplements in future.
\end{abstract}

KEY WORDS: blood; dietary supplement; drug development; gene expression; mRNA.

\section{INTRODUCTION}

Drug candidates are usually selected by various cellfree systems followed by a series of experiments with cultured cells and experimental animals. The final one then moves to clinical trials. However, because of the gap between these preclinical study models and the human body, many drug candidates fail during clinical trials. Some drugs are very effective in cultured cells or animal models, but fail to show any efficacy in humans. Moreover, unexpected severe side effects may happen only in human. Since the clinical trials are extremely expensive and have a potential for life-threatening adverse reactions, the improve-

Part of the study was presented at TriConference in 2007 in San Francisco.

Electronic supplementary material The online version of this article (doi:10.1007/s11095-007-9510-2) contains supplementary material, which is available to authorized users.

${ }^{1}$ Hitachi Chemical Research Center, Inc., 1003 Health Sciences Road, Irvine, California, USA.

${ }^{2}$ Hitachi Chemical Co., Ltd., Hitachi, Japan.

${ }^{3}$ Inflammatory Bowel Disease Center, Cedars-Sinai Medical Center, Los Angeles, California, USA.

${ }^{4}$ Fucoidan Research Institute, Fukuoka, Japan.

${ }^{5}$ Hitachi Ltd., Hitachi General Hospital, Hitachi, Japan.

${ }^{6}$ To whom correspondence should be addressed. (e-mail: mmitsuhashi@ HCRcenter.com) ment in preclinical studies is clearly one of the main priorities of pharmaceutical research.

Although early stage drug candidates cannot be administered to humans, it is technically and ethically feasible to mix these drugs in human whole blood to evaluate the efficacy and toxicity. It is particularly reasonable if such drugs are planned to be administered intravenously or expected blood levels are predetermined by mathematical models or animal experiments. Because in vitro assays are safe, a large number of healthy and disease donors can be recruited. Unfortunately, such blood experiments do not analyze drug actions on organs or whole body. However, it is at least useful to identify the effect on leukocytes. Thus, the methodology is primarily applicable to anti-inflammatory and immunomodulatory drugs. It may be useful for other drugs to assess adverse effects on leukocytes, because leukocyte toxicity is occasionally fatal. Moreover, leukocytes may be used as a surrogate marker for various diseases.

Typically, mononuclear leukocytes are isolated from human whole blood, suspended in culture media, incubated in a $\mathrm{CO}_{2}$ incubator for a couple of days to several days with drugs, and various drug-related protein syntheses and biological functions are determined. However, the assay condition is no longer physiological due to a lack of communication among different cell populations, oxygen supply from erythrocytes, as well as complex interactions with plasma proteins and other components. Secondary reactions may happen during the lengthy 
incubation period. In order to avoid these problems, our group recently reported a novel assay system, in which human whole blood was stimulated with appropriate drugs in vitro for less than $4 \mathrm{~h}$, and drug-induced mRNA expression was quantified $(1,2)$. The use of whole blood and a short incubation period are very physiological and considered as ex vivo.

Although the induction of mRNA does not always correspond to protein synthesis and subsequent biological and clinical outcomes due to altered splicing, post-translational modification, co-activation of inhibitory cascades, etc., the induction of appropriate mRNA under ex vivo condition in each patient will be an interesting research model for drug development. Especially, this ex vivo assay may become a powerful tool for the analysis of individual-to-individual variations, which is a foundation of tailored medicine, and is difficult to assess by current preclinical studies. In order to emphasize the value of ex vivo assay, various applications are demonstrated in this study, although each system should be validated clinically using appropriate drug targets.

\section{MATERIALS AND METHODS}

\section{Materials}

Anti-T cell receptor (TCR) monoclonal antibody (IgG1 $\kappa)$, control mouse IgG1 $\kappa$ (BioLegend, San Diego, CA, USA), GSK$3 \mathrm{~b}$ inhibitor I, JNK inhibitor II, JAK inhibitor I, Aloisine A, (Calbiochem, San Diego, CA, USA), fucoidan (Dr. Fucoidan V-MAX, Ventuno, Fukuoka, Japan) were obtained from designated suppliers. Reagents for mRNA analysis were the same as our previous publication (1). All other chemicals were purchased from Sigma-Aldrich (St. Louis, MO, USA).

\section{Blood Sampling}

Heparinized whole blood samples were obtained from Apex Research Institute (Tustin, CA, USA), Hitachi, Ltd., Hitachi General Hospital (Hitachi, Japan), Cedars-Sinai Medical Center (Los Angeles, CA, USA), and Salon de Mediage Shirogane (Fukuoka, Japan) after respective Institutional Review Board (IRB) approvals. Blood samples were stored at $4^{\circ} \mathrm{C}$, and stimulated with appropriate drugs in the same day of blood draw. After each drug treatment, blood samples were stored frozen at $-80^{\circ} \mathrm{C}$.

\section{Primers and Probes}

Polymerase chain reaction (PCR) primers and TaqMan probes were designed by Primer Express (Applied Biosystem, Foster City, CA, USA) and HYBsimulator (RNAture, Irvine, CA, USA) $(3,4)$ (Supplemental Table 1). Oligonucleotides were synthesized by IDT (Coralville, IA, USA), Tsukuba Oligo Service (Tsukuba, Japan), Nippon EGT (Toyama, Japan), and Hokkaido System Science (Sapporo, Japan).

\section{Blood Stimulation}

In eight-well strip microtubes, $1.2-1.4 \mu \mathrm{l}$ of $50 \times$ concentrations of drugs or controls [phosphate buffered saline (PBS) or dimethysulfoxide (DMSO)] were added, and stored at $-20^{\circ} \mathrm{C}$ until use. About $60-70 \mu \mathrm{l}$ of blood samples was added into each well in triplicate, and incubated at $37^{\circ} \mathrm{C}$ for $2-4 \mathrm{~h}$ with cap closed. In some experiments (Figs. 2, 3 and 8), inhibitors were added $30 \mathrm{~min}-1 \mathrm{~h}$ prior to the stimulations. After each treatment, $50 \mu \mathrm{l}$ of whole blood was transferred to filterplates as described in the next section.

\section{mRNA Quantification}

The quantity of mRNA was determined by the method we developed previously $(1,2)$. In brief, $50 \mu \mathrm{l}$ of blood samples were applied to 96-well filterplates to trap leukocytes. Sixty microliters of lysis buffer containing a cocktail of specific reverse primers was applied to the filterplates, and the resultant cell lysates were transferred to oligo(dT)immobilized microplates (GenePlate, RNAture) $(5,6)$ for poly $(\mathrm{A})^{+}$mRNA purification. The cDNA was directly synthesized in $30 \mu \mathrm{l}$ solutions at each well: specific primerprimed cDNA in the liquid phase and oligo(dT)-primed cDNA in the solid phase (1). Four microliters of undiluted (Figs. 1, 2, 3, 4, 5, 7 and 8) or diluted (Fig. 6) cDNA solution was used for TaqMan PCR (Figs. 3, 4, 5 and 8) (7) or SYBR Green PCR (Figs. 1, 2, 6 and 7) (8) using iTaqSYBR master mix (BioRad, Hercules, CA, USA), as described previously $(1,2)$. Each gene was amplified individually. The cycle threshold $(\mathrm{Ct})$, which was the cycle of PCR to generate certain amounts of PCR products (fluorescence), was determined using analytical software (SDS, Applied Biosystems). In order to maintain the assay specificity of SYBR Green PCR, 1× RT buffer was used as negative controls to confirm no primer dimer was generated under this PCR condition. Moreover, the
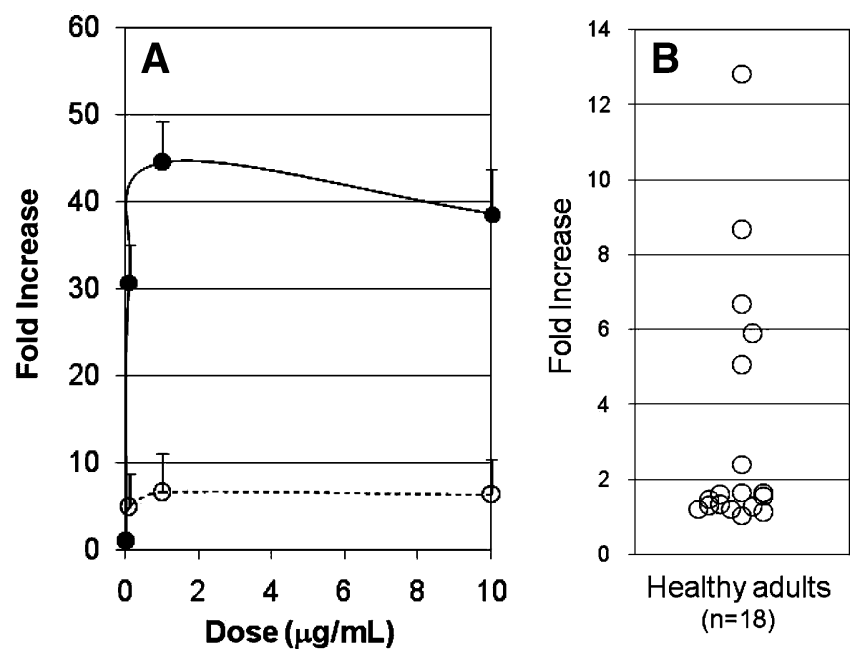

Fig. 1. T-cell receptor (TCR)-mediated TNF $\alpha$ mRNA expression in human whole blood. A Fifty microliters of heparinized human whole blood was stimulated in triplicate with various concentrations of antiTCR monoclonal antibody (filled circle) or control IgG (open circle) for $2 \mathrm{~h}$ at $37^{\circ} \mathrm{C}$. The TNF $\alpha$ mRNA was then quantified using SYBRgreen PCR, and the fold increase was calculated using the values of solvent (PBS) as control as described in "Materials and Methods". Each data point is the mean \pm standard deviation (SD) from triplicate aliquots of whole blood. B Summary of 18 healthy adult volunteers using $10 \mu \mathrm{g} / \mathrm{ml}$ each of anti-TCR or control IgG. Because control IgG increased the levels of mRNA (A, open circle), the fold increase was calculated using the values of the same concentrations of control $\mathrm{IgG}$ in the subpanel $\mathbf{B}$. Each data point is the mean value from each individual. 


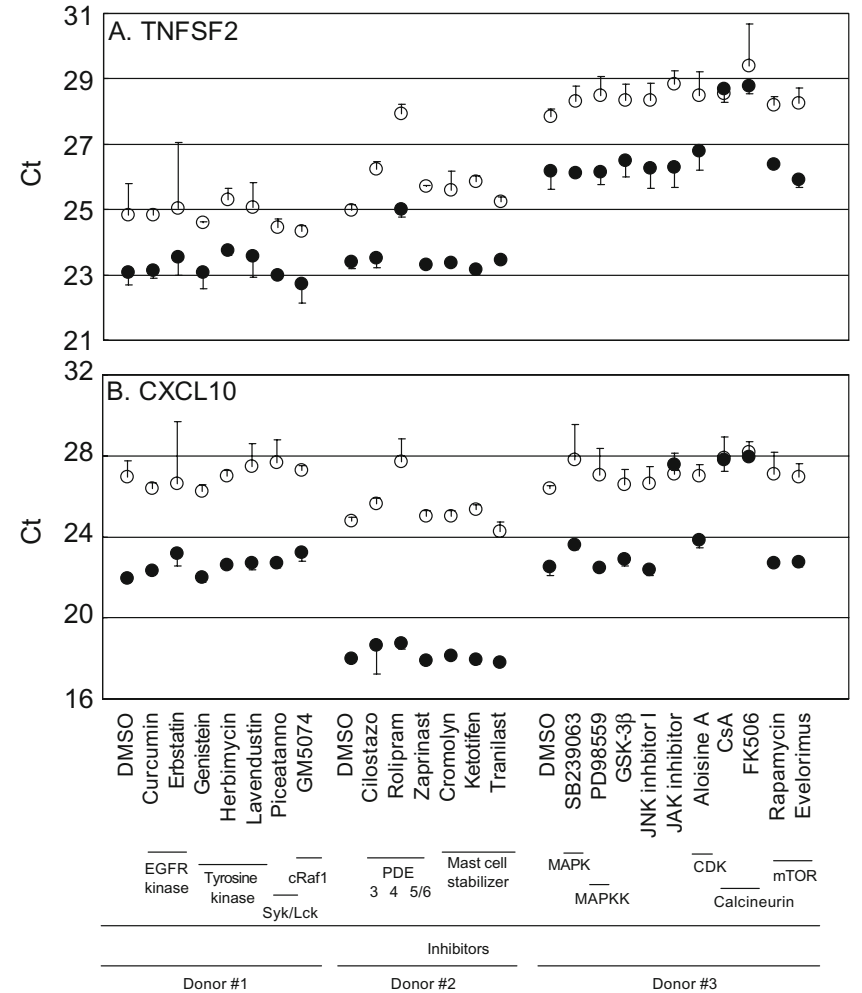

Fig. 2. Ex vivo compound screening. Using three donors (1-3), $50 \mu \mathrm{l}$ each of heparinized human whole blood was exposed to various drugs (all $10 \mu \mathrm{M}$ in the final concentrations) for $1 \mathrm{~h}$ in triplicate, then stimulated with $10 \mu \mathrm{g} / \mathrm{ml}$ each of anti-TCR (filled circle) or control IgG (open circle) for an additional $2 \mathrm{~h}$. TNF $\alpha$ (A) and CXCL10 (B) mRNA were quantified as described above. The $y$-axis was the $\mathrm{Ct}$, the cycle of PCR to generate certain amounts of PCR products: the smaller $\mathrm{Ct}$ values mean higher expression, and $1 \mathrm{Ct}$ difference means double or one half in quantity. Each data point is the mean \pm SD from triplicate aliquots of whole blood. melting curve was analyzed in each case to confirm that PCR signals were derived from a single PCR product. We also measured standard curves during PCR using known concentrations $\left(1-10^{6}\right.$ copies/well) of synthetic DNA templates, and found that the efficiency of PCR was always close to $100 \%$ under this PCR condition (annealing for 30 s plus extension for 1min) regardless of target species, because tenfold differences of the amounts of templates were approximately 3.3 in $\Delta \mathrm{Ct}\left(2^{\wedge} 3.3=9.8\right.$ folds $)$. More importantly, the amplification slope of the samples was always identical to that of corresponding templates, suggesting that PCR efficiency was conserved between the templates and samples. Thus, the $\mathrm{Ct}$ values of drug-treated triplicate samples were subtracted by the mean $\mathrm{Ct}$ values of control samples to calculate $\Delta \mathrm{Ct}$, and the fold increase was calculated as $2^{\wedge}(-\Delta \mathrm{Ct})$.

\section{RESULTS AND DISCUSSION}

\section{Identification of Respondents and Non-Respondents}

Prediction of respondents and non-respondents against specific drug treatment is critically important for clinical trials. Once non-respondents are identified before drug treatment, such patients will be excluded from the clinical trial to improve the chance of success of the study. Figure 1 was an initial attempt to this field. Mouse monoclonal antibody IgG1 $\kappa$ against human $\alpha / \beta \mathrm{T}$ cell receptor (TCR) induced tumor necrosis factor $\alpha(\mathrm{TNF} \alpha)$ mRNA in a dose dependent manner, when antibody was incubated in heparinized human whole blood for only $2 \mathrm{~h}$ (Fig. 1A, filled circle). Control mouse IgG1 $\kappa$ also increased TNF $\alpha$ mRNA (Fig. 1A, open circle), although the values were much smaller than those of antiTCR. Thus, fold increase was calculated by using the same concentrations of control mouse IgG (Fig. 1B). TCR activation was known to produce TNF $\alpha$ (9), however, we found that approximately one third of healthy population increased
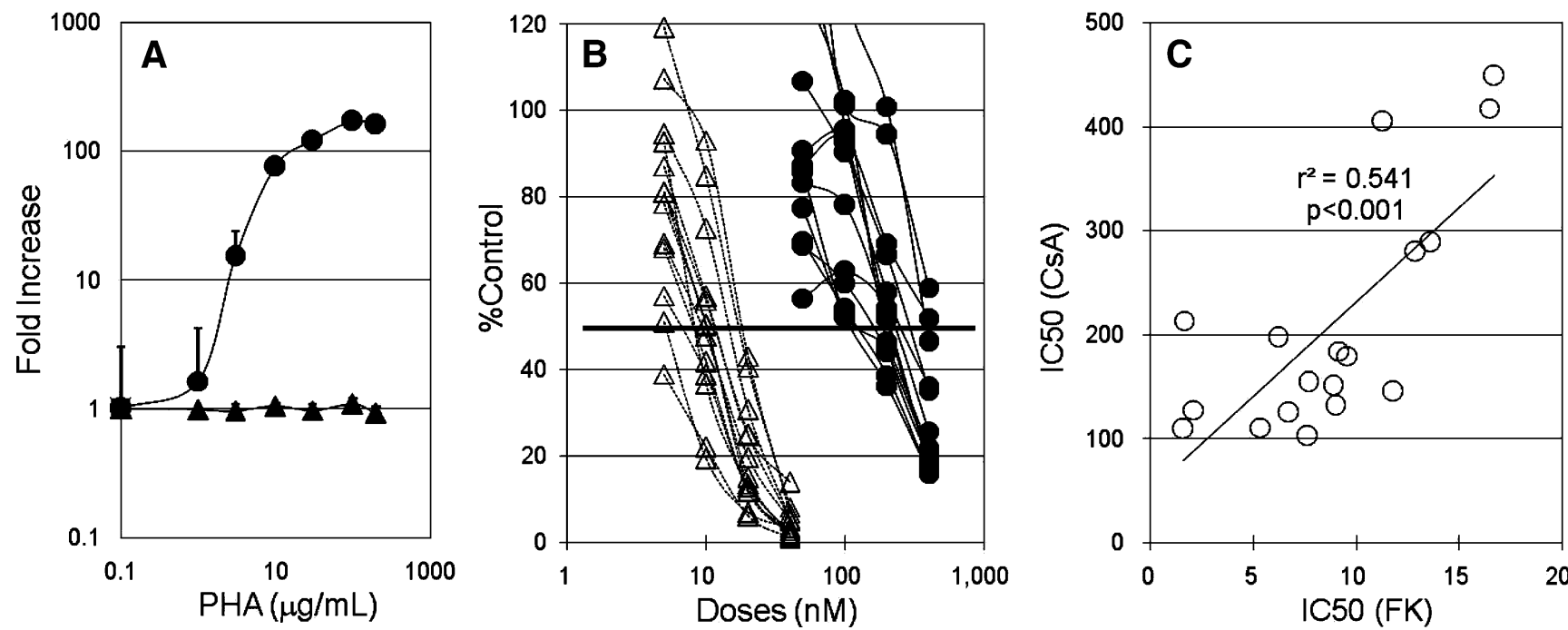

Fig. 3. PHA-induced IL-2 mRNA expression in human whole blood. A Blood samples were stimulated with various concentrations of phytohemagglutinin-P (PHA) or control (PBS) for $1 \mathrm{~h}$, then IL-2 (filled circle) and $\beta$-actin (filled triangle) mRNA were quantified using TaqMan as described in the Methods. B Blood samples were pretreated with 50-400 nM of CsA (filled circle) or 5-40 nM of FK506 (open triangle) for $1 \mathrm{~h}$, then stimulated with $30 \mu \mathrm{g} / \mathrm{ml}$ of PHA or control PBS at $37^{\circ} \mathrm{C}$ for another $1 \mathrm{~h}$. The $\%$ Control was calculated by using the values of PBS (no PHA) and PHA (no inhibitor) as 0 and 100\%, respectively, for each individual. C The IC 50 of both CsA ( $y$-axis) and FK506 $\left(x\right.$-axis) was plotted. Each data point is the mean $\pm \mathrm{SD}(\mathbf{A})$ or mean $(\mathbf{B}, \mathbf{C})$ from each individual. The regression line, $r^{2}$, and $p$ value are shown in the subpanel $\mathbf{C}$. 


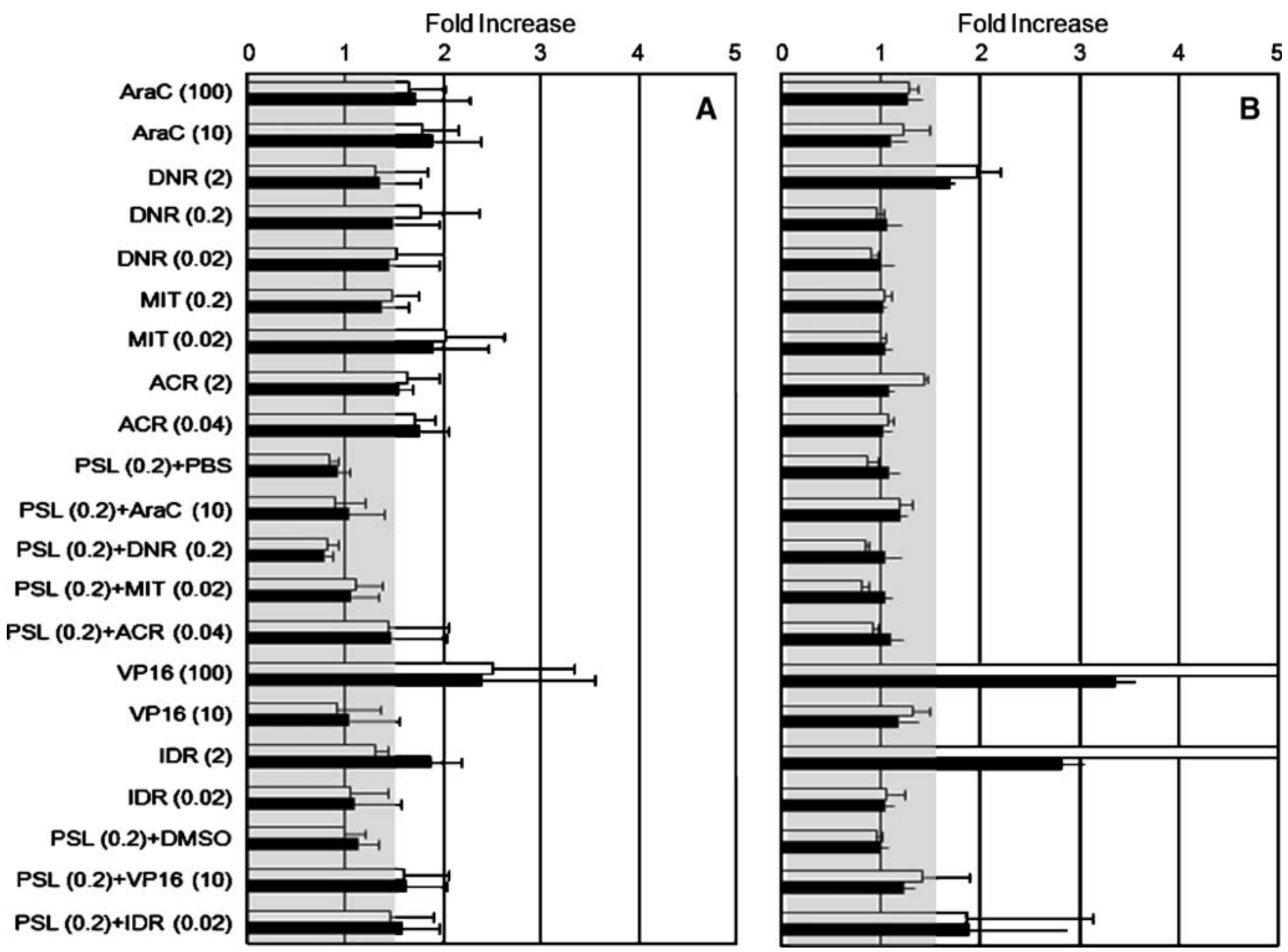

Fig. 4. Drug-induced p21 and PUMA mRNA expression in whole blood from leukemia patients. Peripheral blood samples from 64 years old male patient with acute myelogenous leukemia (AML) (A) and 53 years old male patient with acute promyeocytic leukemia (APL) (B) were mixed with various anti-leukemia drugs at $37^{\circ} \mathrm{C}$ for $4 \mathrm{~h}$, then p21 (open square) and PUMA (filled square) mRNA were quantified using TaqMan as described in the "Materials and Methods". The population of leukemic cells was 96 (A) and 94\% (B), respectively. Each data point is the mean $\pm \mathrm{SD}$ from triplicate aliquots of whole blood. Hashed areas are negative with $<1.5$ fold increase. Drugs used were cytarabine $($ AraC), daunorubicin $(D N R)$, mitoxantrone $(M I T)$, aclarubicin $(A C R)$, and etoposide $(V P 16)$, idarubicin $(I D R)$, and predonisone $(P S L)$ with designated dose (in parentheses) in $\mu \mathrm{M}$.

TNF $\alpha$ mRNA, whereas remaining two thirds failed to respond, even the highest concentrations of antibodies $(10 \mu \mathrm{g} / \mathrm{ml})$ were used (Fig. 1B). The $y$-axis of Fig. 1A and B is different, because the control values were solvent (PBS) (A) and control IgG at each time point (B), respectively. This is the first report showing ex vivo respondents and non-respondents for TCR-mediated TNF $\alpha$ mRNA induction, although its clinical implication is not yet characterized.

TCR is a heterodimer molecule of immunoglobulin-like $\alpha$ and $\beta$ chains, and is associated with 4 molecules of CD3 $(\gamma, \delta$, and $2 \varepsilon), 2 \zeta$ chains, and CD8 (homo- or hetrodimer of $\alpha$ and $\beta$ chains) to form the TCR-CD3 complex. TCR $\alpha / \beta$ chains are the molecules to recognize the specific structure of the target to elicit various immunological reactions. Figure 1 was a model system to assess TCR functionality by activating TCR $\alpha / \beta$ chains with agonistic anti-TCR antibody. The components of TCR-CD3 complex have multiple transcript variants as well as various genetic polymorphisms (10-13). TCR-CD3 complex is also known to activate various kinases $(14,15)$, and these kinases have multiple transcript variants and genetic polymorphisms, too (16). Thus, altered functions of TCR shown in Fig. 1 may happen by genetic alterations of any of these genes. Alternatively, non-respondents may have some inhibitor in plasma. However, it is time consuming, labor-intensive, and expensive to identify genetic polymorphisms of these genes as well as discovery of unknown plasma factors in each human subject. The identification of functional respondents and non-respondents under physiological ex vivo condition may be useful to classify the patient population for clinical trials of immunomodulatory drugs and vaccines, where TCR plays a crucial biological function.

\section{Ex Vivo Compound Screening}

Drug candidate compounds are usually screened by cellfree system or cultured cells. Figure 2 is a new concept of $e x$ vivo screening directly using human whole blood. Each blood sample was exposed to various drugs $1 \mathrm{~h}$ prior to anti-TCR stimulation, then TNF $\alpha$ (Fig. 2A) mRNA was quantified as described above. Furthermore, additional CXCL10 cytokine 

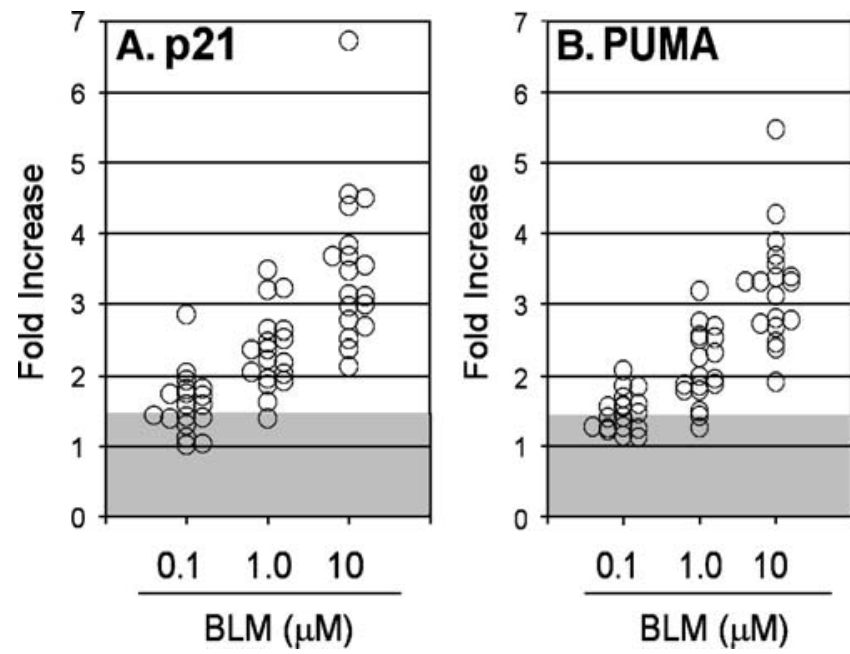

Fig. 5. Bleomycin $(B L M)$-induced $\mathrm{p} 21$ and PUMA mRNA expression in human whole blood. Blood samples from 18 healthy adult volunteers were stimulated with $0-10 \mu \mathrm{M}$ BLM for $4 \mathrm{~h}$, then p21 (A) and PUMA (B) mRNA were quantified using TaqMan as described in the "Materials and Methods". Each data point is the mean from triplicate whole blood samples in each individual. Hashed areas are considered as negative response with $<1.5$ fold increase.

mRNA was also quantified (Fig. 2B). Blood volume used for each volunteer was less than $3 \mathrm{ml}[8$ (7 drugs and 1 control $) \times$ 2 (control $\mathrm{IgG}$ and anti-TCR) $\times 3$ (triplicate) $\times 60 \mu \mathrm{l}]$. The $y$-axis was the $\mathrm{Ct}$, the cycle of PCR to generate certain amounts of PCR products: the smaller $\mathrm{Ct}$ values mean higher expression, and $1 \mathrm{Ct}$ difference means double or one half in quantity. In order to screen a wide spectrum of compounds, each donor was treated with different drugs. Three donors (all respondents) showed different expression levels of TNF $\alpha$ and CXCL10 mRNA (Fig. 2, open circle), and cilostazol (Fig. 2A) and rolipram (Fig. 2A,B) decreased the basal expression levels of these mRNA (Fig. 2, open circle). AntiTCR antibody induced TNF $\alpha$ and CXCL10 mRNA (Fig. 2, filled circle), with the $\mathrm{Ct}$ difference approximately 2 (=4 fold increase) and 4-6 (=16-64 fold increase) for $\mathrm{TNF} \alpha$ and CXCL10 mRNA, respectively, in these 3 donors. However, cyclosporine (CsA) and tacrolimus (FK506) completely eliminated the induction of $\mathrm{TNF} \alpha \mathrm{mRNA}$ (Fig. 2A, filled circle). Interestingly, additional JAK inhibitor I also inhibited CXCL10 mRNA induction (Fig. 2B, filled circle). These results were reasonable, because the effects of cyclosporine $(17,18)$ and JAK kinase inhibitor (19) were reported previously.

As screening assay, $10 \mu \mathrm{M}$ was used for all drugs in Fig. 2, because $10 \mu \mathrm{M}$ is a highest achievable blood level for many drugs. Once candidates are identified, the same assay platform can be used to draw an $\mathrm{IC}_{50}$ as well as the analysis of individual-to-individual variations among large number of healthy adults and appropriate disease patients. When the compounds are selected for clinical trials, the same assay can be used to identify respondents from the study subjects as discussed above. The ex vivo drug screening shown in Fig. 2 will be a valuable tool for drug development, including various kinase inhibitors for inflammation, autoimmune diseases, transplantation, etc. The concept of ex vivo drug screening is expandable beyond anti-TCR-induced TNF $\alpha$ and CXCL10 mRNA by introducing various other stimulations and corresponding target mRNA.

\section{Determination of Individually Optimized Doses}

Unlike Fig. 1, many drugs show dose dependent reactions, and study subjects will be classified into low- and highrespondents. For low respondents, drug doses should be increased to induce appropriate clinical responses, whereas drug doses should be decreased for high-respondents to eliminate unwanted side effects. Determination of individually optimized doses is not only useful for clinical practice as a tailored medicine, but also applicable to study design of clinical trials in future. Figure 3 demonstrated an example of such dose-dependent ex vivo analysis. Phytohemagglutinin-P (PHA) increased IL2 mRNA in a dose dependent manner (Fig. 3A, filled circle), whereas control $\beta$-actin mRNA was unchanged (Fig. 3A, filled triangle). Pretreatment with cyclosporine (CsA) and tacrolimus (FK506) inhibited PHAinduced IL2 mRNA expression in a dose dependent manner (Fig. 3B), with an $\mathrm{IC}_{50}$ of $100-450 \mathrm{nM}$ and $1-18 \mathrm{nM}$ for CsA and FK, respectively (Fig. 3C). The results were similar to those reported by other groups $(20,21)$, however, our method was more practical in terms of blood volume, throughput, and reproducibility.

The range of $\mathrm{IC}_{50}$ varies 5-20 folds, suggesting that drug doses may be determined individually by assessing $\mathrm{IC}_{50}$ for each patient. The practice of therapeutic drug monitoring

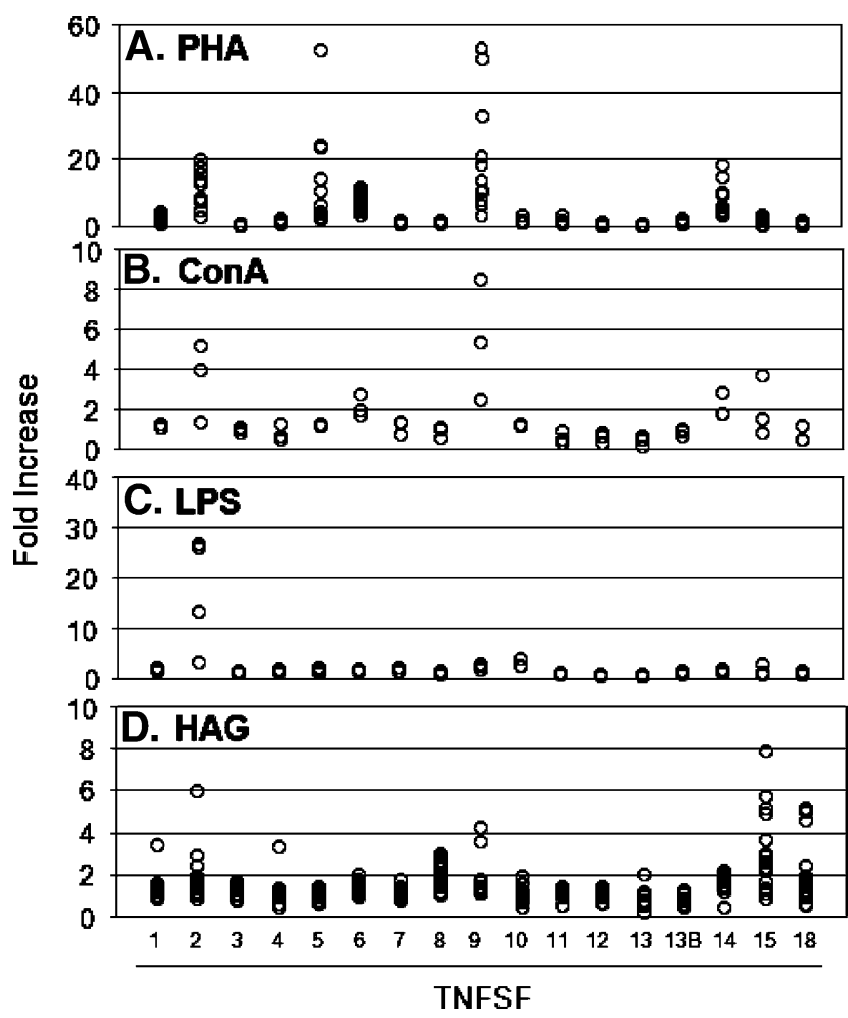

Fig. 6. Screening of tumor necrosis factor superfamily (TNFSF) mRNA. Blood samples were stimulated with either $100 \mu \mathrm{g} / \mathrm{ml}$ each of PHA (A, $n=12)$ or convanavalin A $(\mathbf{B}, n=3), 10 \mu \mathrm{g} / \mathrm{ml}$ lipopolysaccharide (LPS) (C, $n=4)$, or $200 \mu \mathrm{g} / \mathrm{ml}$ of heat aggregated human IgG (HAG) $(\mathbf{D}, n=20)$ at $37^{\circ} \mathrm{C}$ for $4 \mathrm{~h}$, then all members of TNFSF mRNA were quantified using SYBRgreen PCR and fold increase was calculated by using the values of solvent (PBS) as control, as described in the Methods. The cDNA was diluted 1:4 with water, and used for PCR. TNFSF10, 11, 18 showed some primer dimers. Each data point is the mean from triplicate whole blood samples in each individual. 

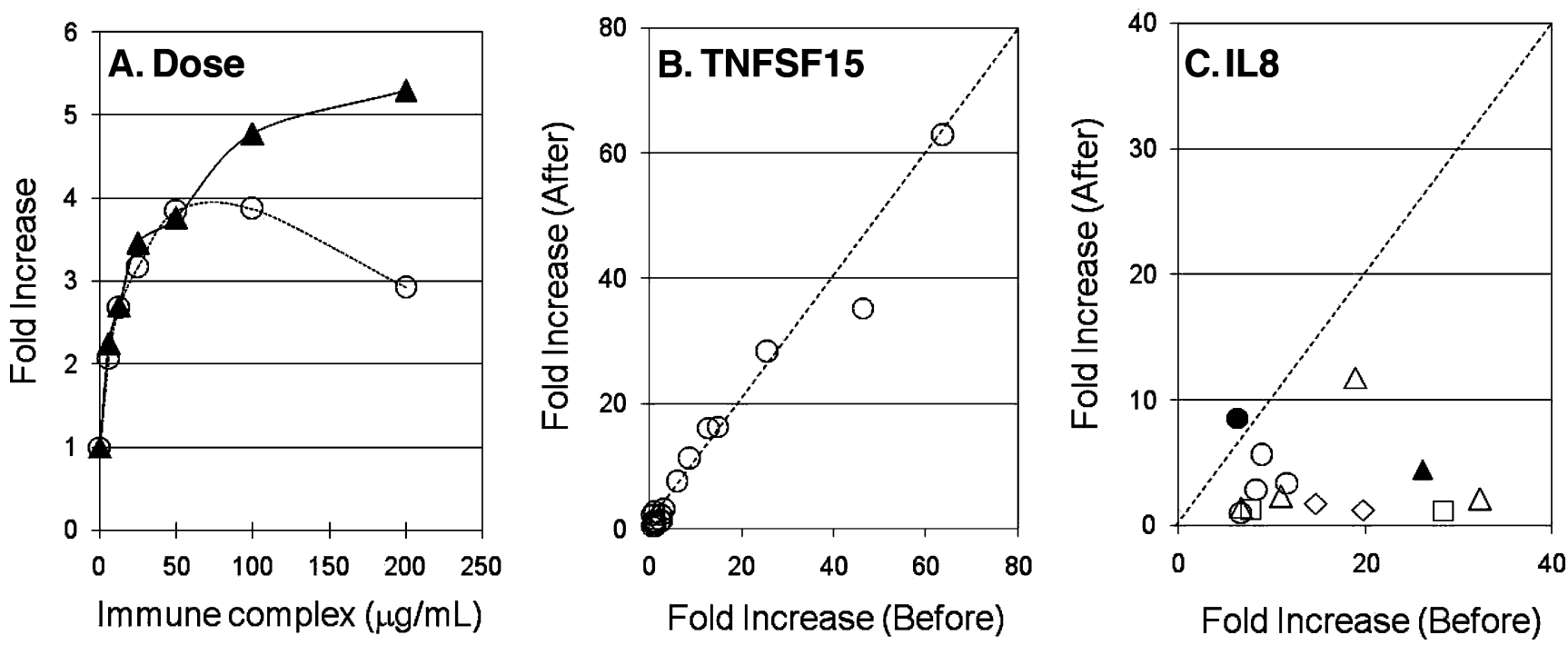

Fig. 7. Effects of fucoidan ingestion on HAG-induced TNFSF15 and IL8 mRNA expression. A Blood samples were stimulated with various concentrations of HAG, then TNFSF15 (open circle) or IL8 (filled triangle) were quantified. Each data point is the mean from triplicate aliquots of whole blood from a single individual. B, C Blood samples were obtained before and $4 \mathrm{~h}$ after $50 \mathrm{ml}$ of fuoidan $(2,550 \mathrm{mg})$ ingestion. These samples were then stimulated with $100 \mu \mathrm{g} / \mathrm{ml}$ of HAG at $37^{\circ} \mathrm{C}$ for $4 \mathrm{~h}$, and TNFSF15 (B) and IL8 (C) mRNA were quantified using SYBRgreen PCR as described in the Methods. Each data point is the mean from each individual. In C, each symbol (open circle, open triangle, open square, and open diamond) represents the same individual tested two to four times with more than 1 week interval.

(TDM) was introduced many years ago when new technologies allowed us to determine the levels of drug concentrations in serum or plasma. Functional variations determined by the ex vivo analysis will be a new addition to TDM in future.

Although high respondents to CsA were also high respondents to FK506, variation existed among individuals (Fig. 3C). This may indicate the possibility of drug preference between CsA and FK506. This is reasonable because CsA and FK506 are acting against different molecular targets [cyclophilin for CsA (22) and FK506 binding protein for FK506 (23)], although both inhibit calcineurin.

\section{Drug-to-Drug Comparison}

When a new drug is introduced to the market, it is required to show advantages of such drug against existing ones, if similar drugs are available. Using whole blood samples from leukemia patients, where more than $96 \%$
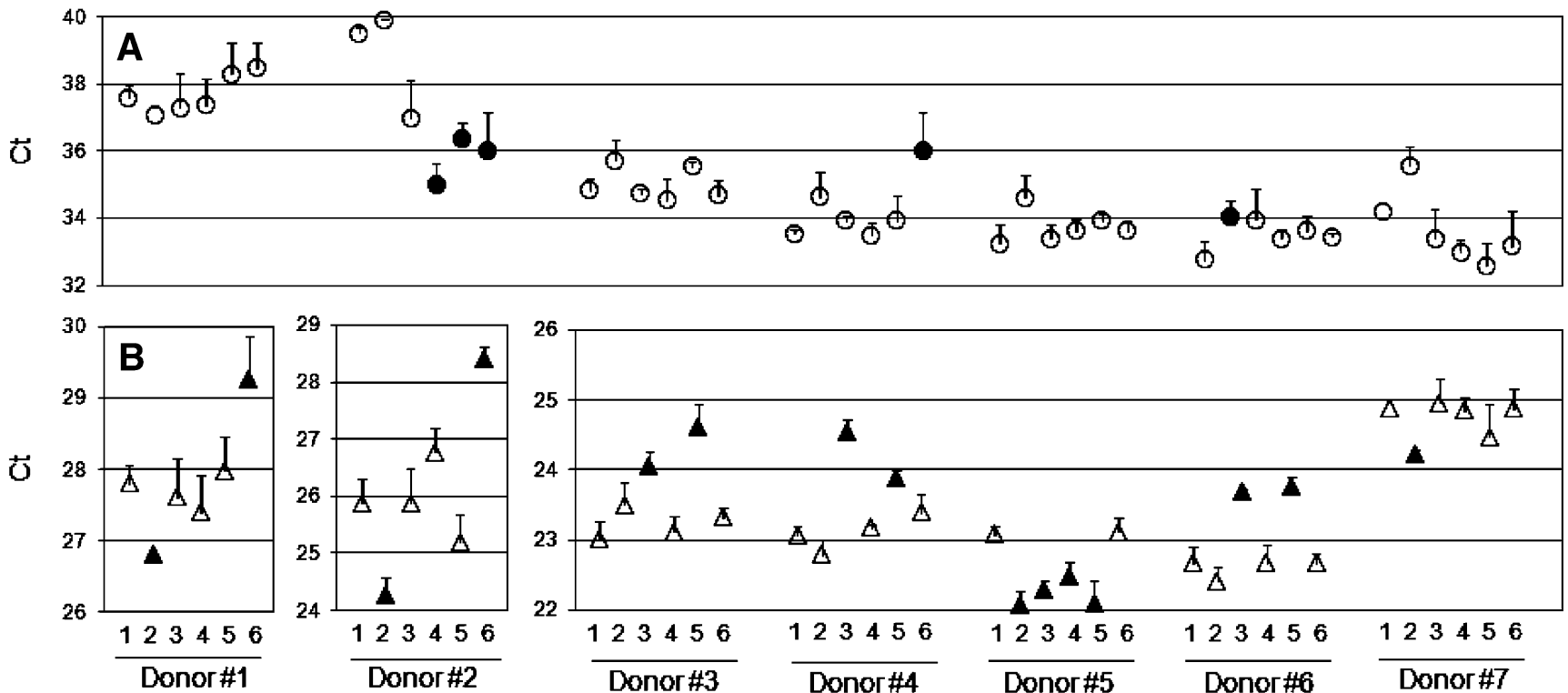

Fig. 8. Effects of various dietary supplements on PHA-induced IL2 mRNA expression. Blood samples from seven healthy adult volunteers (Donor 1-7) were preincubated with various dietary supplements for $1 \mathrm{~h}$, then PHA $(100 \mu \mathrm{g} / \mathrm{ml})$-induced IL2 mRNA was quantitated as described in the "Materials and Methods" and Fig. 3. Each symbol represents the Ct values of control (PBS) (open circle, filled circle) or PHA stimulation (open triangle, filled triangle), respectively. Each data point is the mean \pm SD from triplicate aliquots of whole blood. Filled circle and filled triangle indicated statistical significance $<0.05 .1$ Control, 2 vitamin $\mathrm{A}(0.1 \mu \mathrm{M}), 3$ vitamin $\mathrm{C}(10 \mu \mathrm{g} / \mathrm{ml}), 4$ epigallocatechin gallate (green tea) $(10 \mu \mathrm{M}), 5$ genistein (soybean) $(2 \mu \mathrm{M})$, and 6 curcumin (turmeric) $(200 \mathrm{nM})$. 
(Fig. 4A) or 94\% (Fig. 4B) of leukocytes were leukemic, drug-induced p21 (Fig. 4, open square) and PUMA (Fig. 4, filled square) mRNA were measured. The p21 (cyclindependent kinase inhibitor 1A, CDKN1A) (24) and PUMA (p53 upregulated modulator of apoptosis) (25-27) were known to be induced transcriptionally by 553 when DNA was damaged by genotoxic drugs. In other words, p21 is responsible for cell cycle arrest, and PUMA is pro-apoptotic. Thus, the analysis of these two genes may correspond to cytostatic and cytocidal effects of drugs, similar to bacteriostatic and bacteriocidal effects of antibiotics. As shown in Fig. 4, preferable drugs were different between these patients (MIT, ACR, VP16 for A, DNR, VP16, IDR for B) in ex vivo condition. The comparison of experimental drug candidates with clinically available drugs in whole blood under physiological ex vivo condition will be an interesting model for the development of anti-leukemia drugs. Since the number of leukocytes is high in leukemia in many cases, many drug candidates can be analyzed simultaneously. Moreover, as shown in Fig. 4, where the effect of predonisone (PSL) were also analyzed, different class of drug candidates can be assessed whether they boost the effects of existing drugs, or reverse drug resistance.

\section{Assessment of Leukocyte Toxicity}

Although leukocyte toxicity is not a major problem for anti-leukemia drugs, it becomes a huge problem for anticancer drugs for solid tumors, because it sometimes becomes fatal. Unfortunately, this side effect is unpredictable. Figure 5 is an example, where $0-10 \mu \mathrm{M}$ bleomycin (BLM) were used to stimulate whole blood and p21 and PUMA mRNA were quantified under the identical condition to that of Fig. 4. As shown in Fig. 5, the responses were dose dependent for both p21 and PUMA, and all individuals showed more than 1.9 fold increase at $10 \mu \mathrm{M}$ BLM. Interestingly, approximately half [56\% (A) and 44\% (B)] of healthy individuals responded to $0.1 \mu \mathrm{M}$ BLM to induce p21 and PUMA mRNA, respectively, whereas the other half did not. Among them, one individual failed to induce both p21 and PUMA mRNA even at $1 \mu \mathrm{M}$ of BLM. The results of Fig. 5 do not directly indicate the predictability of leukocyte toxicity, because toxic BLM is not allowed to be administered to healthy individuals. However, the existence of p21 and PUMA respondents at low concentrations of BLM may provide additional considerations for the development of chemotherapeutic drugs.

\section{Discovery of Molecular Targets}

In Fig. 3, PHA-induced IL-2 mRNA was quantified, because the system was previously reported $(20,21)$. Then, the remaining cDNA was further used to screen various mRNA. Figure 6 illustrates the results of the screening of tumor necrosis factor superfamily (TNFSF) mRNA. As shown in Fig. 6A, PHA induced TNFSF2 (=TNF $\alpha), 5$ (=CD40 ligand), 6 (=Fas ligand), 9, and 14 (=LIGHT, CD258). B-cell stimulator lectin, concanavalin A (ConA) induced TNFSF2, 6, 9, and 14 mRNA, but not TNFSF5 mRNA (Fig. 6B). Lipopolysaccharide (LPS), which is known to activate Tolllike receptor-2 (28) and 4 (29) induced mainly TNFSF2
mRNA (Fig. 6C). Moreover, the classic model of immune complex, heat aggregated IgG (HAG) (30) induced different classes of TNFSF, such as TNFSF8 (CD30 ligand, CD153) and 15 (=TL1A) as well as TNFSF2, 9 and 18 in some cases (Fig. 6D). Since human genome and mRNA sequences are available, new molecular targets can be discovered by ex vivo screening as shown in Fig. 6.

HAG-induced TNFSF mRNA may be an interesting clinical research model of antibody medicines, because therapeutic antibodies will form immune complexes in the body by binding to the target molecules. The immune complexes are known to activate $\mathrm{IgG} F \mathrm{Fc}$ receptors $(\mathrm{Fc} \gamma \mathrm{R})$, and FcyR-mediated functions are consisted of numerous variants, such as 4 subclasses of Fc portions of $\operatorname{IgG}$ ( $\mathrm{IgG} 1-$ 4), multiple classes of Fc $\gamma \mathrm{R}$ [Fc $\gamma$ RIa, b, c (=CD64), Fc $\gamma$ RIIa, b, c (=CD32), and FcyRIIIa and b (=CD16)], different subsets of FcyR-bearing leukocytes, and various inducible cytokines. Moreover, these proteins have multiple transcript variants as well as various genetic polymorphisms $(31,32)$. As shown in Fig. 6D, HAG showed respondents and nonrespondents for each of TNFSF mRNA, and these variations may happen at any of these genes. The individual variations of ex vivo responses shown in Fig. 6D may be a predictable marker for the efficacy and toxicity of antibody medicines.

\section{Assessment of Acute Action of Dietary Supplements}

Although various dietary supplements are used widely, the scientific evidence for their efficacy is not well established. One of problems of dietary supplement research is the lack of scientific and quantitative methods to assess acute actions, because chronic effects are difficult to interpret. Since dietary supplements are freely available, we can analyze the effects of dietary supplements before and after ingestion for each individual. Figure 7 was an example of fucoidan, the sea weed extract used widely in Japan. We collected blood samples before and 4 hours after ingestion of $50 \mathrm{ml}$ of fuoidan $(2,550 \mathrm{mg})$, and analyzed the changes of HAG-induced TNFSF15 and IL8 mRNA induction. As shown in Fig. 7A, HAG $(100 \mu \mathrm{g} / \mathrm{ml})$ induced TNFSF15 and IL8 in a dose dependent manner. Although the levels of HAG-induced TNFSF15 mRNA expression were unchanged between these 2 samples (Fig. 7B), the levels of HAG-induced IL8 mRNA was significantly reduced after fucoidan ingestion (Fig. 7C). The same individuals were tested two to four times (Fig. 7C, each symbol represents the same individual), however, the effects of fucoidan was confirmed every time. Although the biological and clinical meanings of the results are not known at this time, it is surprising to find such significant effects within 4h of ingestion. The ex vivo assay will be sensitive enough to analyze the efficacy of dietary supplements in each individual.

\section{Characterization of Respondents and Non-respondents for Dietary Supplements}

Dietary supplements, such as vitamins, polyphenols, turmeric, etc. are known to induce various biological activities in cultured cells and animals, and some of activities were confirmed by subsequent clinical studies. However, the results of clinical studies are difficult to interpret because of the inconsistency of the results. If respondents and non-respondents 
exist, and the later population is much larger than the former, the standard method of double blind clinical trials is no longer useful. Since the blood levels of active ingredients of dietary supplements were well characterized, simulation will be theoretically possible by incubating whole blood with pure ingredients at designated blood levels. In Fig. 8, human whole blood was pretreated with various ingredients 1 hour prior to PHA-induced IL2 mRNA expression analysis. The concentrations of these ingredients used in Fig. $8(0.1 \mu \mathrm{M}$ vitamin $\mathrm{A}$, $10 \mu \mathrm{g} / \mathrm{ml}$ vitamin $\mathrm{C}, 10 \mu \mathrm{M}$ epigallocatechin gallate (green tea), $2 \mu \mathrm{M}$ genistein (soybean), and $200 \mathrm{nM}$ curcumin (turmeric) were achievable blood levels according to various literature. The effects of dietary supplements were much weaker than active drugs as shown in Figs. 2 and 3, however, some showed significant influences on the basal expression levels of IL2 mRNA as well as PHA-induced IL2 mRNA expression (Fig. 8, filles circle, filled triangle). However, such effects were not identified for all individuals. For example, vitamin A enhanced PHA-induced IL2 mRNA expression in four (Donor 1, 2, 5, 7) out of seven individuals, whereas vitamin $\mathrm{C}$ inhibited the reaction in three cases (Donor 3, 4, 6) (Fig. 8B, filled triangle). The ex vivo simulation was sensitive enough to identify respondents and non-respondents for dietary supplements, although biological and clinical meanings are not clear at this time. However, identification of individual variation will be a useful tool for the clinical studies of diet and dietary supplements in future.

\section{CONCLUSIONS}

The ex vivo analysis is a new concept we introduced recently by using an mRNA quantification platform we developed (1,2). In order to explore the possibility of $e x$ vivo assay, various applications are demonstrated in this study. Although mRNA expression does not always correspond to protein synthesis and subsequent biological and clinical outcomes, identification of appropriate mRNA induction in each individual will be a useful information for the development of drugs and dietary supplements. Since $450 \mathrm{ml}$ of blood can be obtained from a single individual $(450 \mathrm{ml}$ is a unit of blood donation for transfusion in the US), and the $e x$ vivo assay uses as little as $50 \mu \mathrm{l}$ per reaction $(150 \mu \mathrm{l}$ in triplicate for each drug), as many as 3,000 drug compounds can be screened. Thus, we believe that the proposed $e x$ vivo assay will be a new addition to pharmaceutical and dietary supplement research.

\section{ACKNOWLEDGMENTS}

We would like to thank Y. Oka, S. Nemoto, N. Sasaki and staffs of clinical laboratories (Hitachi General Hospital, Hitachi, Japan), Dr. MJ Tonkon, C. Fox, C. McGinty, and J. Haire (Apex Research Institute, Tustin, CA, USA), C. Landers and J. Price (Cedars Sinai Medical Center, Los Angeles, CA, USA), K. Nakamizo (Ventuno, Fukuoka, Japan), K. Otsuka (Kamerica, Torrance, CA, USA), T. Otake, H. Ito, and B. Maekawa (Hitachi Chemical, Tokyo, Japan) for the support of the blood draw and clinical section of this study. We also thank C. Yamamoto (Hitachi Chemical Research Center, Irvine, CA, USA) and I. Perlinski (Perlinski and Company, Laguna Niguel, CA, USA) for the review of English.
Open Access This article is distributed under the terms of the Creative Commons Attribution Noncommercial License which permits any noncommercial use, distribution and reproduction in any medium, provided the original author(s) and source are credited.

\section{REFERENCES}

1. M. Mitsuhashi, S. Tomozawa, K. Endo, and A. Shinagawa. Quantification of mRNA in whole blood by assessing recovery of RNA and efficiency of cDNA synthesis. Clin. Chem 52:634-642 (2006).

2. M. Mitsuhashi. Ex vivo simulation of drug action: Quantification of drug-induced mRNA as a bridge between preclinical and clinical trials. Clin. Chem 53:148-149 (2007).

3. M. Mitsuhashi, et al. Oligonucleotide probe design-a new approach. Nature 367:759-761 (1994).

4. D. Hyndman, et al. Software to determine optimal oligonucleotide sequences based on hybridization simulation data BioTechniques 20:1090-1097 (1996).

5. M. Mitsuhashi, C. Keller, and T. Akitaya. Gene manipulation on plastic plates. Nature 357:519-520 (1992)

6. Y. Hamaguchi, et al. Direct reverse transcription-PCR on oligo (dT)-immobilized polypropylene microplates after capturing total mRNA from crude cell lysates. Clin. Chem 44:2256-2263 (1998).

7. P. M. Holland, et al. Detection of specific polymerase chain reaction product by utilizing the $5^{\prime}-3^{\prime}$ exonuclease activity of Thermus aquaticus DNA polymerase. Proc. Natl. Acad. Sci. U.S A 88:7276-7280 (1991).

8. T. B. Morrison, J. J. Weis, and C. T. Wittwer. Quantification of low-copy transcripts by continuous SYBR Green I monitoring during amplification. Biotechniques 24:954-962 (1998).

9. I. Millet, and N. H. Ruddle. Differential regulation of lymphotoxin (LT), lymphotoxin-beta (LT-beta), and TNF-alpha in murine $\mathrm{T}$ cell clones activated through the TCR. J. Immunol 152:4336-4346 (1994).

10. P. Charmley, et al. Human T-cell receptor CD3-epsilon (CD3E)/ TaqI DNA polymorphism. Nucleic Acids Res 17:2374(1989).

11. U. Malhotra, and P. Concannon. Human T-cell receptor CD3delta (CD3D)/MspI DNA polymorphism. Nucleic Acids Res 17:2373(1989).

12. P. Charmley, et al. Human T-cell receptor CD3-gamma (CD3G)/ MspI DNA polymorphism. Nucleic Acids Res 17:2372 (1989).

13. M. H. Polymeropoulos, H. Xiao, D. S. Rath, and C. R. Merril. Dinucleotide repeat polymorphism at the human MHC class I CD8 alpha-chain (Leu-2/T8) gene. Nucleic Acids Res 19:1718 (1991).

14. S. Gibson, et al. The EMT/ITK/TSK (EMT) tyrosine kinase is activated during TCR signaling: LCK is required for optimal activation of EMT. J. Immunol 156:2716-2722 (1996).

15. J. A. Frearson, and D. R. Alexander. The phosphotyrosine phosphatase SHP-2 participates in a multimeric signaling complex and regulates T cell receptor (TCR) coupling to the Ras/ mitogen-activated protein kinase (MAPK) pathway in Jurkat T cells. J. Exp. Med 187:1417-1426 (1998).

16. S. Nervi, et al. No association between lck gene polymorphisms and protein level in type 1 diabetes. Diabetes 51:3326-3330 (2002).

17. T. Espevik, et al. Inhibition of cytokine production by cyclosporin A and transforming growth factor beta. J. Exp. Med 166:571-576 (1987).

18. A. D. Cristillo, M. J. Macri, and B. E. Bierer. Differential chemokine expression profiles in human peripheral blood $\mathrm{T}$ lymphocytes: dependence on T-cell coreceptor and calcineurin signaling. Blood 101:216-25 (2002).

19. N. Kanda, and S. Watanabe. Prolactin enhances interferon-gammainduced production of CXC ligand 9 (CXCL9), CXCL10, and CXCL11 in human keratinocytes. Endocrinology 148:2317-2325 (2007).

20. T. Giese, et al. Monitoring of NFAT-regulated gene expression in the peripheral blood of allograft recipients: a novel perspective 
toward individually optimized drug doses of cyclosporine A. Transplantation 77:339-344 (2004).

21. C. Hartel, et al. Sensitivity of whole-blood T lymphocytes in individual patients to tacrolimus (FK 506): impact of interleukin2 mRNA expression as surrogate measure of immunosuppressive effect. Clin. Chem 50:141-151 (2004).

22. R. E. Handschumacher, M. W. Harding, J. Rice, R. J. Drugge, and D.W. Speicher. Cyclophilin: a specific cytosolic binding protein for cyclosporin A. Science 226:544-547 (1984).

23. J. J. Siekierka, S. H. Hung, M. Poe, C. S. Lin, and N. H. Sigal. A cytosolic binding protein for the immunosuppressant FK506 has peptidyl-prolyl isomerase activity but is distinct from cyclophilin. Nature 341:755-757 (1989).

24. J. Pines. Cell cycle. p21 inhibits cyclin shock. Nature 369:520-521 (1994).

25. J. Han, et al. Expression of bbc3, a pro-apoptotic BH3-only gene, is regulated by diverse cell death and survival signals. Proc. Natl. Acad. Sci. U.S.A 98:11318-11323 (2001).

26. J. Yu, et al. PUMA induces the rapid apoptosis of colorectal cancer cells. Mol. Cell 7:673-682 (2001).
27. K. Nakano, and K. H. Vousden. PUMA, a novel proapoptotic gene, is induced by p53. Mol. Cell 7:683-694 (2001).

28. R. B. Yang, et al. Toll-like receptor-2 mediates lipopolysaccharide-induced cellular signalling. Nature 395:284-288 (1998).

29. J. C. Chow, D. W. Young, D. T. Golenbock, W. J. Chris, and F. Gusovsky. Toll-like receptor-4 mediates lipopolysaccharideinduced signal transduction. J. Biol. Chem 274:10689-10692 (1999).

30. K. K. Ostreiko, I. A. Tumanova, and Yu. K. Sykulev. Production and characterization of heat-aggregated IgG complexes with predetermined molecular masses: light-scattering study. Immunol. Lett 15:311-316 (1987).

31. J. M. Debets, C. J. Van der Linden, I. E. Dieteren, J. F. Leeuwenberg, and W. A. Buurman. Fc-receptor cross-linking induces rapid secretion of tumor necrosis factor (cachectin) by human peripheral blood monocytes. J. Immunol 141:1197-1201 (1988).

32. B. A. Binstadt, R. S. Geha, and F. A. Bonilla. IgG Fc receptor polymorphisms in human disease: implications for intravenous immunoglobulin therapy. J. Allergy Clin. Immunol 111:697-703 (2003). 